\title{
Efficacy of spontaneous pushing with pursed lips breathing compared with directed pushing in maternal and neonatal outcomes: a clinical trial
}

\author{
Ana Eulina Araujo ${ }^{1}$, Alexandre Delgado ${ }^{1}$, Juliana Netto Maia ${ }^{1}$, Shirley Lima Campos ${ }^{1}$, \\ Caroline Wanderley Souto Ferreira ${ }^{1}$, and Andrea Lemos ${ }^{2}$ \\ ${ }^{1}$ Affiliation not available \\ ${ }^{2}$ Universidade Federal de Pernambuco
}

October 15, 2020

\begin{abstract}
Objective: to evaluate the efficacy of spontaneous pushing with pursed lips breathing compared to directed pushing during the second period of labor in the occurrence of episiotomy. Methods: this is a quasi-randomized clinical trial, with 62 low-risk pregnant women in the second stage of labor. They were randomly allocated in control (CG) ( $\mathrm{n}=31$ ) and intervention (IG) $(\mathrm{n}=31)$ groups. The IG performed spontaneous pushing with pursed lips breathing while the CG was oriented to perform directed pushing associated with Valsalva Maneuver (MV). Results: There was no difference between the groups regarding the occurrence of episiotomy (RR 1,1; 95\% IC 1,0 to 1,2 ). However, there was a decrease in the duration of the maternal pushing by 3.2 minutes (MD 3,2; 95\%CI 1,4 to 5,1) and a difference in maternal anxiety (Md (IQR) IG 46 (35-52), CG 51 (44-56) p:0,049), both favoring the IG. There was no difference in others maternal and neonatal outcomes Conclusions: spontaneous pushing with pursed lips breathing was effective in reducing the duration of the pushing and showed a difference in maternal anxiety, but did not decrease the occurrence of episiotomy, nor did it alter the other studied maternal and neonatal outcomes.
\end{abstract}

\section{Abstract}

Objective: to evaluate the efficacy of spontaneous pushing with pursed lips breathing compared to directed pushing during the second period of labor in the occurrence of episiotomy.

Methods: this is a quasi-randomized clinical trial, with 62 low-risk pregnant women in the second stage of labor. They were randomly allocated in control $(\mathrm{CG})(\mathrm{n}=31)$ and intervention $(\mathrm{IG})(\mathrm{n}=31)$ groups. The IG performed spontaneous pushing with pursed lips breathing while the CG was oriented to perform directed pushing associated with Valsalva Maneuver (MV).

Results: There was no difference between the groups regarding the occurrence of episiotomy (RR 1,1; $95 \% \mathrm{IC} 1,0$ to 1,2$)$. However, there was a decrease in the duration of the maternal pushing by 3.2 minutes (MD 3,2; 95\%CI 1,4 to 5,1) and a difference in maternal anxiety (Md (IQR) IG 46 (35-52), CG 51 (44-56) p:0,049), both favoring the IG. There was no difference in others maternal and neonatal outcomes

Conclusions: spontaneous pushing with pursed lips breathing was effective in reducing the duration of the pushing and showed a difference in maternal anxiety, but did not decrease the occurrence of episiotomy, nor did it alter the other studied maternal and neonatal outcomes.

Brazilian Clinical Trials Registry (ReBEC) under the identifier: RBR-556d22.

Keywords: Labor, Obstetric; Labor Stage, Second; Respiration; Parturition; Spontaneous Pushing. 
Funding statement: the study had no funding body.

\section{Introduction}

Maternal pushing has been discussed for decades, mainly about its relevance in facilitating care in the second stage of labor and maternal and fetal outcomes [1,2]. In 1957, Beynon was the pioneer to compare the pushing done spontaneously with the Valsalva maneuver (VM), leading to a discussion about imposed breathing patterns associated with expulsive efforts and their relationship with the integrity of the pelvic floor. That study initiated a reflection on the importance of changing pushing instructions in obstetric care $[3,4]$.

Directed pushing is common practice in obstetrics worldwide and it is done independently of the maternal desire to carry out the expulsive effort. It is usually associated with a VM sustained for 10 seconds or more and is oriented by third parties [4-6].

Recently in 2018, the World Health Organization (WHO) recommended that women in the expulsive phase of the second stage of labor should be encouraged and supported to follow their desire to push. According to the WHO, qualitative evidence shows that it is important for women to feel in charge of childbirth. The recommendation mentions that due to the absence of evidence of any benefit of imposing directed pushing in the second stage of labor this technique should be avoided by health professionals involved in childbirth care [7].

Spontaneous pushing is carried out naturally by the woman according to the inevitable desire to push down progressively. There is no deep breathing, and it does not initiate right at the beginning of the contraction. Generally, three to five pushings occur starting at the peak of the contraction and mostly happen with open glottis. [4-6].

In this scenario, the idea of using pursed lips breathing arises, which consists of a nasal inspiration followed by a resisted exhalation performed with pursed lips and/or semi-closed teeth, which may occur with or without contraction of the abdominal muscles [8] to facilitate spontaneous pushing during the expulsive effort, but studies are still scarce [2]. There is a study that compared a relaxation technique with breathing exercises, which involved diaphragmatic inspiration, prolonged fractional expiration, and pursed lips breathing with usual care but observed only during the first stage of labor [9].

Several physiological effects are related to pursed lips breathing in several populations, such as improvement of breathing patterns, reduction of dyspnea sensation, global relaxation, positive expiratory pressure maintenance, preventing alveolar collapse while promoting lung deflation [8]. Also, an increase in arterial oxygen pressure and arterial oxygen saturation, decreased carbon dioxide pressure, decreased respiratory rate and increased tidal volume were observed [10], in addition to changes in respiratory mechanics, with greater activation of the abdominal, rib cage and accessory expiratory muscles, and lower diaphragmatic participation, which may be useful to prevent respiratory fatigue $[11,12]$.

Therefore, given the physiological benefits of pursed lips breathing described in the literature, we hypothesize that its use during the second stage of labor may result in better maternal and neonatal outcomes and thus provide the necessary evidence for its guidance.

Given the gaps in knowledge resulting from the high risk of bias in the studies regarding the type of pushing, the present study aimed to evaluate the efficacy of spontaneous pushing with pursed lips breathing when compared to directed pushing on maternal and neonatal outcomes.

\section{Materials and methods}

\subsection{Study design}

This is a quasi-randomized controlled clinical trial that compares a group of parturients who received guidance in the second stage of labor regarding spontaneous pushing with pursed lips breathing to a control group that followed the usual care of the service with directed pushing. 


\subsection{Definitions and Participants}

This study was conducted in a maternity hospital located in Recife, Brazil, from July 2018 to January 2019.

Inclusion criteria were low- and high-risk parturients in the second stage of labor, between 19 and 45 years old and 37 to 42 gestational weeks. Were excluded from the study multiparous women, those with dead fetuses, history of analgesia, induced delivery, or using psychoactive drugs.

\subsection{Interventions}

Researchers applied a checklist to confirm eligibility criteria. If the parturient were eligible, she received information about the study and was invited to participate voluntarily. A Free and Informed Consent Form was requested according to the 466/12 Resolution of the National Health Council.

This research was approved by the Research Ethics Committee of the Federal University of Pernambuco under the registration numbers 2.885.560 and CAEE: 81405717.7.0000.5208 and is also registered in the Brazilian Clinical Trials Registry (ReBEC) under the identifier RBR-556d22. The description of this article followed the international standards recommended by the CONSORT Statement: Updated Guidelines for Reporting Parallel Group Randomised Trials [13].

The study sample for the primary outcome (episiotomy) was calculated considering the results of a metaanalysis [2] that obtained a RR of 0,51 and a control group incidence of $71 \%$ through the Open-epiversion 3.0 software, considering alpha and beta errors of 0,05 and 0,20 , respectively. A $20 \%$ loss to follow-up was anticipated. $95 \%$ confidence level and $80 \%$ power were considered. Therefore, 62 pregnant women were necessary, 31 in the intervention group and 31 in the control group.

Randomization was carried out by placing each patient's name in opaque envelopes that corresponded to either the intervention or the control group and were prepared by an independent researcher not directly involved in the study. The parturients were followed in three moments: right after randomization, one hour and 24 hours after delivery.

In this initial follow-up performed during prepartum and in the delivery room the duration of the second stage of labor and the maternal pushings were measured. Then, the following data were collected: personal and socioeconomic (age, marital status, schooling, family income, and occupation), clinical and obstetric data (weight, height, Body Mass Index - BMI, Systolic Blood Pressure and Diastolic Blood Pressure), postpartum hemorrhage, perineal laceration, episiotomy, gestational age, parity, route of delivery, instrumental delivery, pain, anxiety, maternal fatigue, and satisfaction. Finally, the following neonatal data were collected in the obstetric center or at the joint accommodation: newborn admission to intensive care, hypoxic-ischemic encephalopathy, 5-minute Apgar, and neonatal resuscitation.

After the diagnosis by the hospital team about the complete deletion of the cervix (10 centimeters) and effacement, the volunteer in the intervention group received guidance about the breathing pattern associated with expulsive efforts, based on the spontaneous pushing physiology: pursed lips breathing associated with open glottis in tidal volume, controlled by the woman, along with abdominal muscle efforts and perineal relaxation. The team also used communication strategies providing positive feedback and information about labor evolution and the possible sensations (Figure 1).

The control group followed the usual routine of the service team, with the use of directed pushing, regardless of maternal desire, initiated right after uterine contraction, by guiding the woman to perform a deep inspiration, initiating the effort with closed glottis for 10 seconds or more. The entire pushing protocols are described in Figure 1.

\subsection{Data collection}

Maternal and fetal outcomes were evaluated within the first hour after birth. Pain and maternal satisfaction about the type of pushing were measured with a 10-point visual analog scale (VAS), 0 being absence 
of pain/mild pain and low satisfaction, and 10 meaning moderate to severe pain and high satisfaction, respectively [14].

Anxiety levels were assessed with the Brazilian version of the State-Trait Anxiety Inventory (STAI) [15], already adapted and validated for women in labor [16]. It contains 19 statements, each with four agreement levels, scored from 1 to 4 , with 1 indicating the lowest level of anxiety and 4 the maximal level. This score was reversed for the items that are present with anxiety (items $1,2,4,7,9,10,14,15,18$, 19). With a total score of 19 to 76 points, mild anxiety (19 to 36 points), moderate anxiety (37 to 56 points) and severe anxiety ( 57 to 76 points) were categorized.

To assess maternal fatigue the Maternal Perception of Childbirth Fatigue Questionnaire (MCFQ) was used, consisting of 15 questions, with four agreement levels each, scored from 0 to 4,0 being an indicative response of a minimum degree of fatigue and 4 a maximum degree, being categorized as low fatigue (15-50 points) and high fatigue (51-75 points) [17].

Data about episiotomy, perineal lacerations, instrumental delivery (forceps or vacuum), postpartum hemorrhage (24 hours after delivery) and neonatal data such as the presence of hypoxic-ischemic encephalopathy, the $5^{\text {th }}$ minute Apgar and the newborn's admission to intensive care were collected in medical records. The positions adopted by women in labor have not been reported or established.

\subsection{Data analysis}

The Student's t-test or the Mann-Whitney test were used to compare continuous variables between the groups. To compare categorical variables the Chi-square test or Fisher's exact test was performed.

To determine the effect size of the intervention, continuous variables were expressed as mean and standard deviation, mean difference and 95\% confidence interval, or median and interquartile interval (25\% - $75 \%)$. The categorical variables were expressed in frequency and number of cases. When relevant, the relative risk was calculated. Kolmogorov-Smirnov normality test was performed for data distribution analysis. Perprotocol analysis was performed. $\mathrm{P}<0,05$ was considered for statistical significance.

All data analysis was performed in SigmaPlot 12.0 (Systat Software, Inc., Germany) and IBM SPSS Statistics Software 25.0 (Statistical Package for the Social Sciences).

\section{Results}

During data collection, 786 parturients were evaluated for eligibility, and 210 were randomized into the two groups. However, a total of 148 volunteers were lost, 80 from the intervention group and 68 from the control group, for the following reasons: labor induction and withdrawal from participation (Figure 2). The final sample consisted of 62 patients, 31 in each group. No high-risk pregnant women were included in this study.

The volunteers had a median age of 23 years (IQR IG: 21-28; CG: 19-30) and median gestational age of 39 weeks (IQR IG: 38-40; CG: 38-40), with no difference between the groups regarding maternal, sociodemographic and biological variables (Table 1 ).

No difference was observed for the following neonatal and maternal outcomes: episiotomy, perineal lacerations, duration of the second stage of labor, vaginal, cesarean, or instrumental delivery. There were no cases of postpartum hemorrhage and maternal blood pressure changes. However, there was a 3.2-minute (IG: 3.2 \pm 3.5 ; CG: $6.5 \pm 3.8$; CI95\% 1.4 to $5.1 ; \mathrm{p}<0.001$ ) (Table 2) decrease in the duration of the maternal pushing and lower maternal anxiety in IG (Md (IQR) IG 46 (35-52) CG 51 (44-56) p:0,049). There was no difference in pain, maternal fatigue, and satisfaction (Table 3).

Regarding neonatal outcomes, there were no cases of newborn admission to intensive care, hypoxic-ischemic encephalopathy, Apgar scores lower than 7, and neonatal resuscitation between the groups.

\section{Discussion}


In the results obtained from this study, the spontaneous pushing associated with pursed lips breathing showed no difference for the occurrence of episiotomy, perineal lacerations, the duration of the second stage of labor, vaginal, Cesarean or instrumental delivery, when compared to directed pushing. There were no cases of postpartum hemorrhage and maternal blood pressure changes. However, there was a decrease in the duration of the maternal pushing and a difference in maternal anxiety in the spontaneous pushing group with pursed lips breathing. There was no difference in pain, fatigue, and maternal satisfaction. There were no events related to neonatal outcomes.

The expulsive stage is considered a strong indicator of long-term impairments of pelvic floor and bladder functions [18-22]. However, the present study showed no difference between the groups regarding episiotomy and perineal lacerations associated with the type of pushing, confirming the findings of a meta-analysis [2] comprised of five studies with 2.320 women in which no difference in episiotomy occurrence was observed RR 0,95; CI95\% 0,87 to 1,04) as well as for grade 3 or 4 perineal lacerations (RR 0,94; CI95\% 0,78 to 1,14), as analyzed from seven studies with 2.775 women. These data differ from a prospective cohort and a randomized clinical trial $[22,23]$ published after that meta-analysis, which evidenced the association of directed pushing with VM maintained for 10 seconds or more with a significant increase in the number of episiotomies and grade 3 and 4 perineal lacerations [24].

It was hypothesized that the possible effects of pursed lips breathing would interfere directly in the pelvic floor muscles, since this exercise causes a change in the recruitment pattern of respiratory muscles, increasing the recruitment of accessory muscles of the chest wall and the activity of the abdominal muscles throughout the respiratory cycle, simultaneously decreasing the recruitment of diaphragmatic and pelvic floor muscles [25]. Therefore, one can conclude that the effect of that breathing pattern on pelvic floor muscles depends on the intensity of the abdominal muscles recruitment as the maneuver can be performed with a strong contraction of those muscles, depending on professional instruction and stimulus.

The intensity of abdominal muscles contraction will influence intra-abdominal pressure (IAP). When contracting the abdominal muscles, the diaphragm rises, while the pelvic floor muscles move downwards [26]. Also, in situations of strong abdominal muscles contractions, the diaphragm moves upwards, and the increase in IAP induces a contraction of the pelvic floor muscles [27].

Therefore, considering that the pelvic floor muscles do not contract by themselves, but in cooperation with the muscles around the abdominal area [28], we believe that this reasoning could be applied during the execution of pursed lips breathing, thus promoting the same effects, which can help the pushing in caudal direction [29].

The duration of the second stage of labor has been discussed as an important aspect of parturition, as the ideal duration for this stage is sought. The factors that influence the duration of this stage have been studied for the development of recommendations such as the breathing patterns used during expulsive efforts. There is no consensus yet regarding these practices and the ideal duration of this stage. It is estimated, though, that the longer the duration, the greater the maternal-fetal repercussion $[18,24,30]$.

When evaluating the duration of the second stage of labor there were no differences regarding the type of pushing. These findings are similar to those of an aforementioned meta-analysis [2], which included six studies comparing the duration of that stage of labor associated with spontaneous pushing or with the directed pushing with VM. A total of 667 nulliparous women were evaluated, initially showing no difference in expulsive stage duration (MD 10.26 minutes; CI95\%: -1,12 to 21,64). However, after sensitivity analysis, due to inadequate randomization, based on four studies with 494 women, a decrease in the duration of the expulsive stage with directed pushing was observed (MD:17,62; CI95\%: 5,28 to 29,95). Those findings were yet considered inconsistent as they presented high heterogeneity due to methodological and statistical limitations, thus with a high association to random effects for those affirmations.

In another retrospective cohort study [22] conducted in Australia with 19.212 women a longer duration of the second stage of labor was observed for those who used the directed pushing when compared to the spontaneous pushing, that duration being 14.4 minutes (95\%CI 12.0-16.8) for the nulliparous and 8.0 minutes $(95 \% \mathrm{CI}$ 
6.8-9.2) for the multiparous. A randomized clinical trial [18] with 108 women corroborated the findings of the previous meta-analysis [2] regarding the expulsive period duration. Therefore, the discussion about the effect of the type of pushing on the duration of the expulsive period persists.

The duration of maternal pushing is another aspect that can influence maternal-fetal well-being and perineal integrity [31-33]. The women from our study who performed spontaneous pushing with pursed lips breathing showed a 3.2-minute reduction of the expulsive effort. This finding was similar to those of a meta-analysis [2] that analyzed pushing duration based on two studies with a total of 169 women, observing a decrease of 9.76 minutes (MD -9.76 minutes; $95 \%$ CI -19.54 to 0.02). However, after sensitivity analysis based on a study with 69 women, there was a 15-minute reduction in pushing duration for the spontaneous pushing group (MD -15,22 minutes; $95 \%$ CI -21,64 to -8,80). This analysis was based on one study only with a small sample and therefore should be interpreted with caution [2].

Favoring the discussion about the importance of these outcomes, a cohort [34] that analyzed 57.267 deliveries concluded that a maternal pushing more than 30 minutes long during the second stage of labor, and an expulsive stage more than one hour long are the potential factors for maternal and neonatal morbidities, especially postpartum hemorrhage, as well as uterine atony and cervical and perineal lacerations. This study recommends caution regarding obstetric interventions and better analyzing expectant management during the second stage of labor.

There was no difference between the types of pushing and delivery route or the need for instrumentalization. These data are similar to those found in the literature [2]. When analyzing research on pain, anxiety, and maternal fatigue in the second period of labor there is no consistent evidence about a direct association with the type of pushing. It is understood, however, that those outcomes might influence labor progression, as the stress generated by these sensations result in a greater release of catecholamines, fatty acids, and lactate, which can reduce the effectiveness of uterine contractions, possibly leading to prolonged labor, and consequently to dystocia, instrumentalization, higher post-partum hemorrhage risk, fetal distress and negative labor experience for the woman $[32,34,35]$.

In our study, women who carried out spontaneous pushing with pursed lips breathing showed lower anxiety levels when compared to those on the control group. This result was seen because the more encouraged to do directed pushing with VM, the less physiological was the labor, thus increasing maternal distress following the release of hormones such as catecholamines and adrenaline, responsible for increasing maternal anxiety $[16]$.

The type of maternal pushing did not influence neonatal outcomes in this study. This was expected since the apnea duration was lower than what is found in literature, which estimates that 7 to 8 seconds of apnea with high intrathoracic and abdominal pressures can already interfere in uteroplacental oxygen delivery, which can lead to fetal distress [36-43].

Not collecting data about the posture adopted by the parturient can be cited as a limitation of this study. That data can influence both the duration of the second stage of labor and the pushing. External conditions related to the hospital environment, such as noise or collective hospitalization, may have distracted the parturient's focus on spontaneous pushing and breathing patterns orientations. Nevertheless, it is important to emphasize that there was no follow-up by the doulas of the service in any of the groups.

It is noteworthy that the exercises were performed by physical therapists. As we know, the individual must be well instructed by the professional to properly perform the technique. The technique is limited to 3 to 5 breaths, as prolonging it causes fatigue of the respiratory muscles and significantly lower levels of carbon dioxide, potentially leading to a decrease in perfusion to the brain, causing syncope. Without the proper use of pursed lips breathing an individual could exacerbate air and carbon dioxide retention [44].

In conclusion, spontaneous pushing with pursed lips breathing was not effective in reducing episiotomy. However, pushing duration decreased by 3.2 minutes, also showing a difference in maternal anxiety. This result may indicate its use for emotional control when compared to directed pushing. As an implication 
for physical therapy practice, these findings may signal an attitude in decision-making about guiding the breathing pattern in the expulsive stage.

\section{Disclosure of interests}

The study has no financial and religious interest, however, there is personal interest to know if the use of the spontaneous pushing with pursed lips breathing improves maternal and neonatal outcomes, as well as, in the political scope of building public and scientific interest, in improving conduct during labor.

\section{Author Contributions}

AEA and AD contributed to project development, data collection and analysis, and manuscript writing. AL, JNM, SLC and CWSF contributed to project development and manuscript writing.

\section{Details of ethics approval}

Research Ethics Committee of the Federal University of Pernambuco under the registration numbers 2.885.560 and CAEE: 81405717.7.0000.5208

\section{Funding}

The study had no funding body.

\section{Acknowledgments}

The authors thank the parturients who participated in the study.

\section{Conflicts of Interest}

The authors have no conflicts of interest.References

[1] G.D. Read. An Outline of the Conduct of Physiological Labor** Presented by invitation, at a meeting of the Maternity Center Association, New York Academy of Medicine, January 17, 1947. Am J Obstet Gynecol, [s.l.], 702 (1947) 710 https://doi.org/10.1016/S0002-9378(16)39605-3.

[2] A. Lemos, M.M. Amorim, A. Dornelas de Andrade, A.I. de Souza, J.E. Cabral Filho, J.B. Correia, Pushing/bearing down methods for the second stage of labour. Cochrane Database Syst Rev, 1 (2017) 107 https://doi.org/10.1002/14651858.CD009124.

[3] C.L. Beynon, The normal second stage of labour. A Plea for Reform in Its Conduct, J Obstet Gynaecol Br Emp, 815 (1957) 820 http://dx.doi.org/10.1111/j.1471-0528.1957.tb08483.x.

[4] R. Caldeyron - Barcia, The influence of maternal bearing- down efforts during the second stage on fetal well- being. Birth Fam J, 17(1979) 21 https://doi.org/10.1111/j.1523-536X.1979.tb01298.x

[5] M. Prins, J. Boxem, C. Lucas, E. Hutton, Effect of spontaneous pushing versus Valsalva pushing in the second stage of labour on mother and fetus: a systematic review of randomised trials. BJOG, 662 (2011) 670 http://dx.doi.org/10.1111/j.1471-0528.2011.02910.x.

[6] Z. Ahmadi, S. Torkzahrani, F. Roosta, N. Shakeri, Z. Mhmoodi. Effect of Breathing Technique of Blowing on the Extent of Damage to the Perineum at the Moment of Delivery: A Randomized Clinical Trial. Iran J Nurs Midwifery Res. 62 (2017) 66. http://dx.doi.org/10.4103/1735-9066.202071.

[7] WHO recommendations: intrapartum care for a positive childbirth experience. Geneva: World Health Organization; 2018. Licence: CC BY-NC-SA 3.0 IGO.

[8] VATWANI, Archana. Pursed Lip Breathing Exercise to Reduce Shortness of Breath. Archives of physical medicine and rehabilitation, v. 100, n. 1, p. 189-190, 2019. DOI:https://doi.org/10.1016/j.apmr.2018.05.005

[9] A. Boaviagem, E. Melo Junior, L. Lubambo, P. Sousa, C. Aragão, S. Albuquerque, A. Lemos The effectiveness of breathing patterns to control maternal anxiety during the first period of labor: 
A randomized controlled clinical trial. Complementary Therapies in Clinical Practice. 30 (2016) 35. http://dx.doi.org/10.1016/j.ctcp.2016.11.004

[10] Tiep BL, Burns M, Kao D, Madison R, Herrera J. Pursed lips breathing training using ear oximetry. Chest. 1986;90:218-21.8.

[11] Breslin EH. The pattern of respiratory muscle recruitment during pursed-lip breathing. Chest. 1992;101:75-8.

[12] ROSSI, Renata Claudino; PASTRE, Carlos Marcelo; RAMOS, Ercy Mara Cípulo and VANDERLEI, Luiz Carlos Marques. A respiração frenolabial na doença pulmonar obstrutiva crônica: revisão da literatura. Fisioter. Pesqui. [online]. 2012, vol.19, n.3 [cited 2020-06-10], pp.282-289.

[13] K.F. Schulz, D.G Altman, D. Moher. CONSORT 2010 statement: updated guidelines for reporting parallel group randomised trials. BMC medicine. 2010;8(1):18.

[14] Gift AG. Visual analogue scales: measurement of subjective phenomena. Nurs Res 1989; 38:286-8.

[15] A.M.B. Biaggio, L. Natalício, C.D. Spielberger, Desenvolvimento da forma experimental em português do Inventário de Ansiedade Traço-Estado (IDATE) de Spielberger. Arq Bras Psicol Aplic, 31 (1977) 44

[16] Delgado A, Freire AB, Wanderley ELS, Lemos A. Validity and Consistency Analysis of the STAI S-Anxiety Scale for 536 Pregnant Women during Labor. RBGO Ginecol Obstet. 38(11); 2016: 531-537. http://dx.doi.org/10.1055/s-0036-1593894

[17] Delgado A, Oliveira PDNF, Góes PSA, Lemos A. Development and analysis of measurement properties of the "Maternal Perception of Childbirth Fatigue Questionnaire" ' (MCFQ). Braz J Phys Ther. 2019;23(2):125131. https://doi.org/10.1016/j.bjpt.2019.01.004

[18] R.G. Koyucu, N. Demirci. Effects of pushing techniques during the second stage of labor: A randomized controlled trial. Taiwan J Obstet Gynecol 606 (2017) 612. https://doi.org/10.1016/j.tjog.2017.02.005

[19] H.P. Dietz, M.J. Bennet, The effect of childbird on pelvic organ mobility. Obstet Gynecol, 223 (2003) 228

[20] H P. Dietz, V. Lanzarone, Levator trauma after vaginal delivery. Obstet Gynecol, 707 (2005) 712 http://doi:10.1097/01.AOG.0000178779.62181.01.

[21] A.M. Thompson, Maternal behaviour during spontaneous and directed pushing in the second stage of labour. J Adv Nurs, 1027(1995) 1034. https://doi.org/10.1111/j.1365-2648.1995.tb03101.x

[22] N. Lee, et al., Maternal and neonatal outcomes from a comparison of spontaneous and directed pushing in second stage, Women Birth (2018), https://doi.org/10.1016/j.wombi.2018.10.005.

[23] J. Schaffer, et al., A randomized trial of the effects of coached vs uncoached maternal pushing during the second stage of labor on postpartum pelvic floor structure and function. Am J Obstet Gynecol 1692 (2005) 1696. https://doi.org/10.1016/j.ajog.2004.11.043

[24] Y. Zipori et al., The impact of extending the second stage of labor to prevent primary cesarean delivery on maternal and neonatal outcomes. Am J Obstet Gynecol. 191(2019). https://doi:10.1016/j.ajog.2018.10.028.

[25] Fregonezi GA, Resqueti VR, Guell Rous R.Pursed Lips Breathing. Archivos de Bronconeumologia. 2004; 40(6):279-282.

26- Bartelink DL: The role of abdominal pressure in relieving the pressure on the lumbar intervertebral discs. J Bone Joint Surg Br, 1957, 39-B: 718-725. [PubMed] [Google Scholar]

[27] Cresswell AG, Grundström H, Thorstensson A: Observations on intra-abdominal pressure and patterns of abdominal intra-muscular activity in man. Acta Physiol Scand, 1992, 144: 409-418. [PubMed] [Google Scholar] 
[28] Sapsford RR, Hodges PW: Contraction of the pelvic floor muscles during abdominal maneuvers. Arch Phys Med Rehabil, 2001, 82: 1081-1088.

[29] Park H, HAN D. The effect of the correlation between the contraction of the pelvic floor muscles and diaphragmatic motion during breathing. J Phys Ther Sci. 2015 Jul; 27(7): 2113-2115.

[30] G. Yildirim, N.K. Beji, Effects of pushing techniques in birth on mother and fetus: a randomized study. Birth, 25 (2008) 30. https://doi.org/10.1111/j.1523-536X.2007.00208.x

[31] W.A. Grobman et al., Association of the Duration of Active Pushing With Obstetric Outcomes. Obstet Gynecol. 667 (2016) 673. https://doi:10.1097/AOG.0000000000001354.

[32] K.J. Aderhold, J.E. Roberts, Phases of second stage labor. Four descriptive case studies, J Nurse Midwifery, 267 (1991) 275 http://dx.doi.org/10.1016/0091-2182(91)90041-m.

[33] C.M. Sampselle, S. Hines, Spontaneous pushing during birth: relationship to perineal outcomes. J NurseMidw. 36 (1999) 39 https://doi.org/10.1016/S0091-2182(98)00070-6

[34] L.C Moya-Jiménez, M.L. Sánchez-Ferrer, E. Adoamnei, J. Mendiola. New approach to the evaluation of perineal measurements to predict the likelihood of the need for an episiotomy. Int Urogynecol J. 815 (2019) 821. https://doi.org/10.1007/s00192-018-3745-9.

[35] E. Looft,, M. Simic,, M. Ahlberg, J.M. Snowden, Y.M. Cheng. Duration of Second Stage of Labour at Term and Pushing Time: Risk Factors for Postpartum Haemorrhage. Paediatr. Perinat. Epidemiol. 126 (2017) 133 https://doi.org/10.1111/ppe.12344.

[36] E. Noble, Controversies in maternal effort during labour and delivery. J Nurse Midwifery, 13(1981)22 https://doi.org/10.1016/0091-2182(81)90003-3

[37] B.M. Yli, et al. How does the duration of active pushing in labor affect neonatal outcomes ?* J Perinat Med. 171 (2011) 178. http://doi:10.1515/JPM.2011.126.

[38] P. Catala, C. Peñacoba, J Carmona, D Marin. Do maternal personality variables influence childbirth satisfaction? A longitudinal study in low-risk pregnancies. Women Health. 1 (2019) 15. https://doi:10.1080/03630242.2019.1613473.

[39] J.M. Roberts, C.B.P. González, C. Sampselle Why Do Supportive Birth Attendants Become Directive of Maternal Bearing-Down Efforts in Second-Stage Labor? J Midwifery Womens Health, 134 (2007) 141 http://dx.doi.org/10.1016/j.jmwh.2006.11.005.

[40] J.E. Roberts, The "push" for evidence: management of the second stage. J Midwifery Women's Health. 2 (2002) 15 https://doi.org/10.1016/S1526-9523(01)00233-1

[41] G. Bassel, S.G. Humayun, G.F. Mark, Maternal bearing down efforts - another fetal risk? Obstet Gynecol, 39 (1980) 41 https://doi.org/10.1097/00132582-198103000-00011

[42] V.M. Roemer, K. Harms, H. Buess, T.J. Horvath, Response of fetal acid- base balance to during of second stage of labour, Int J Gyanecol Obstet. 455 (1976) 471 https://doi.org/10.1002/j.1879-3479.1976.tb00084.x

[43] A. Lemos, E. Dean, A.D Andrade. The Valsalva maneuver duration during labor expulsive stage: repercussions on the maternal and neonatal birth condition. Rev Bras Fisioter. 66 (2011) 72. http://dx.doi.org/10.1590/S1413-35552011000100012.

[44] Nguyen J, Duong H. Pursed-lip Breathing. [Updated 2019 Sep 9]. In: StatPearls [Internet]. Treasure Island (FL): StatPearls Publishing; 2020 Jan-. Available from: https://www.ncbi.nlm.nih.gov/books/NBK545289/

Group

Description

Intervention Group (Spontaneous pushing with pursed lips breathing)

Nasal inspiration followed by a resisted expiration, 
Figure 1. Assistance protocol for pushing guidance in control and intervention groups.

Table 1. Maternal sociodemographic and biological characteristics of the groups that performed spontaneous pushing with pursed lips breathing and directed pushing during the second stage of labor.

\begin{tabular}{|c|c|}
\hline & IG $(n=31)$ \\
\hline$\overline{\text { VARIABLES }}$ & Median (IQR25-75) \\
\hline Age $_{(\text {years })}$ & $23(21-28)$ \\
\hline Height $(\mathrm{cm})$ & $162(159-167)$ \\
\hline Weight $_{(\mathrm{kg})}$ & $69,9(63,5-79,5)$ \\
\hline BMI $_{(\mathrm{kg} / \mathrm{m} 2)}$ & $26,6(24,5-30,3)$ \\
\hline Gestational age $_{(\text {weeks })}$ & $39(38-40)$ \\
\hline VARIABLES & $\mathrm{N}(\%)$ \\
\hline \multicolumn{2}{|l|}{ Parity } \\
\hline Nuliparous & $0(0)$ \\
\hline Primiparous & $7(22)$ \\
\hline Multiparous & $24(78)$ \\
\hline \multicolumn{2}{|l|}{ Origin } \\
\hline Recife and metropolitan region & $30(97)$ \\
\hline Other regions & $1(3)$ \\
\hline \multicolumn{2}{|l|}{ Marital status } \\
\hline Single & $19(61)$ \\
\hline Married & $8(26)$ \\
\hline Divorced & $1(3)$ \\
\hline Widowed & $0(0)$ \\
\hline Civil partnership & $3(10)$ \\
\hline \multicolumn{2}{|l|}{ Education (years) } \\
\hline 1 to 3 & $0(0)$ \\
\hline 4 to 7 & $27(87)$ \\
\hline 8 to 11 & $4(13)$ \\
\hline$>12$ & $0(0)$ \\
\hline \multicolumn{2}{|l|}{ Per capita income $(\mathrm{MW})$} \\
\hline$<1$ & $8(26)$ \\
\hline 1 to 3 & $23(74)$ \\
\hline 4 to 6 & $0(0)$ \\
\hline$>7$ & $0(0)$ \\
\hline \multicolumn{2}{|l|}{ Occupation } \\
\hline Housewife & $24(77)$ \\
\hline Other & $7(23)$ \\
\hline IQR - interquartile interval; N - number of cases. MW - Minimum wage R $\$ 954,00$ (2018) & IQR - interquartile interval; \\
\hline
\end{tabular}

Table 2. Maternal outcomes of parturients submitted to spontaneous pushing with pursed lips or to directed pushing.

MATERNAL VARIABLES

Duration of the second stage of labor (minutes) 
MATERNAL VARIABLES

Duration of maternal pushing (minutes)

Episiotomy

Perineal laceration

Vaginal delivery

Instrumental delivery

Cesarean section

Postpartum hemorrhage

Maternal blood pressure

Oxytocin

SD, standard deviation; MD, difference in means; N, number of cases; RR, Relative Risk; ${ }_{95 \%} \mathrm{CI}, 95 \%$ Confidence Interval.

Table 3. Pain, fatigue, anxiety, and maternal satisfaction scores for the spontaneous pushing with pursed lips breathing and the directed pushings groups during the second stage of labor.

\begin{tabular}{ll}
\hline VARIABLES & IG \\
\hline VAS PAIN SCORE & $\mathbf{N}(\%)$ \\
No pain / mild pain & $0(0)$ \\
Moderate pain / severe pain & $31(100)$ \\
MATERNAL FATIGUE SCORE & $\mathbf{N}(\%)$ \\
Low fatigue & $27(87)$ \\
High fatigue & $4(13)$ \\
IDATE ANXIETY SCORE & $\mathbf{N}(\%)$ \\
Mild anxiety & $4(12)$ \\
Moderate anxiety & $25(81)$ \\
High anxiety & $2(7)$ \\
MATERNAL SATISFACTION SCORE & $\mathbf{N}(\%)$ \\
Low satisfaction & $4(13)$ \\
High satisfaction & $27(87)$ \\
IG, intervention group; CG, control group. 1 Mann-Whitney test & IG, intervention group; CG, control group. 1 Mann-Wh \\
\hline
\end{tabular}

